\section{Social and nonsocial sources of attraction in rats*}

\author{
BIBB LATANE and CAROL WERNER \\ Ohio State University, Columbus, Ohio 43210
}

Rats were more attracted to other rats than to a number of nonsocial objects, each chosen as possible sources of physical satisfaction, such as warmth, shade, secondary reinforcement, novelty, etc. Social attraction may be independent of such mundane needs and may require social satisfactions such as mutual activity and interaction.

When allowed to interact freely in an open environment, rats are quite attracted to one another, staying much closer together than by chance and spending up to two-thirds of their time in direct physical contact (Cappell \& Latané, 1969; Eckman, Meltzer, \& Latané, 1969; Latané, 1969; Latané, Cappell, \& Joy, 1970; Latané \& Glass, 1968). Why are rats so attracted to one another? What motives are satisfied by social approach? Before postulating specifically social motives, it might be well to look for nonsocial satisfactions rats can provide for one another.

Rats may be attracted to each other to find warmth, secondary reinforcement, shade, physical contact, or novelty, among other things. If any one of these satisfactions is to account for social attraction, it should be possible to show that rats are attracted to nonsocial objects which also provide these attractions. The present experiment compares rats' attraction to other rats, with their attraction to a variety of nonsocial stimulus objects selected to satisfy different nonsocial motives.

SUBJECTS AND APPARATUS

Fifty experimentally naive male Sprague-Dawley albino rats were 37 days old at the time of the study. The animals were received in the laboratory 7 days prior to testing and were individually housed for the duration of the experiment, with Purina laboratory pellets and water available ad lib from a food hopper and water bottle suspended outside their cages.

The open field used for measuring attraction has been described in greater detail elsewhere (Latane, 1969). Essentially, it is a circular wooden field, $4 \mathrm{ft}$ in diam, in which the rats can roam freely; its uniform banality prevents attraction to any particular area of the field. The field is

\footnotetext{
* This research was supported by National Science Foundation Grant GS 2292. We thank P. Gillig. D. Henderson, D. Kile, D. Markiewicz, M. Saks, R. Sloan, and D. Walton for their help. Request reprints from B. Latané $404 \mathrm{C}$ West $17 \mathrm{th}$ Avenue, Columbus. Ohio 43210 .
}

painted white, and numbered grid markings on the floor are used to record the location of the rat and the stimulus objects. Uniform illumination is provided by a shaded $60-\mathrm{W}$ bulb suspended above the center and slightly below the rim of the field. In a darkened room, only the field interior is lighted and the $O$ is shielded from the rat's vision.

\section{EXPERIMENTAL DESIGN}

Rats were tested once a day for 6 days with one of the stimulus objects described below. The objects were selected because they represented specific qualities of a rat which might hold some attraction for other rats. They were placed near the wall of the open field to be easily accessible without impeding exploration around the perimeter.

\section{Warm Bottle}

One quality which may attract rats to one another is their body warmth. If so, we might expect rats to be attracted to other warm objects, especially once they have had a chance to become familiar with them. In the present experiment, eight rats were tested for 6 days with a standard 8-oz six-sided Pyrex Evenflo baby bottle filled with $100^{\circ} \mathrm{F}$ water immediately prior to each use. The bottle, with nipple attached, was placed on its side near the wall of the field. By the end of the 5 -min test period, the water temperature usually dropped to about $97^{\circ} \mathrm{F}$. Even at this temperature, the bottle feels pleasantly warm to the at the .05 level by Duncan's multiple range test. $+p<.001$. human hand. Rats were unable to obtain water from the bottle.

\section{Food Pellets}

Rats may be secondary reinforcers to each other, since they obtain primary reinforcements (such as food) in the presence of other rats (Antonitis \& Sher, 1952). If secondary reinforcement leads to attraction, objects which have been associated with primary reinforcement, such as food itself, should also be attractive. In the present experiment, eight rats were tested for 6 days with 12 pellets of Purina laboratory chow enclosed in a heavy wire mesh frog (ordinarily used to stabilize floral arrangements). The frog was a 1-in.-high 3-in.-diam hemispherical container made of $1 / 2-i n$. wire mesh comparable to that separating rats from their food in their home cages. Although all rats were on an ad lib food schedule and presumably not greatly hungry, foodpellets should still be a powerful source of secondary reinforcement, since they have a strong association with eating.

\section{Dark Rathole}

Rats are nocturnal animals who tend to prefer the dark to the bright light. Perhaps rats are attracted to each other in bright surroundings because they can use each other to avoid the light. If this is true, rats should also be attracted to nonsocial objects which provide similar protection. In the present experiment, eight rats were tested for 6 days with an 8-in.-long 23/4-in.-i.d. Plexiglas tube, painted black on the outside and placed on its side near the wall of the open field. Rats could enter either end of the tube, sit beside it, crawl over it, or sniff about it.

\section{Clear Rathole}

In addition to seeking shade, rats are also attracted to walls. In previous experiments using the present apparatus, for example, rats have typically spent over $90 \%$ of their time in the outer $50 \%$ of the open field, near the wall. In order to assess the effects of such thigmotaxis,

Table 1

Effects of Stimulus Objects

\begin{tabular}{lcccc}
\hline $\begin{array}{l}\text { Stimulus } \\
\text { Object }\end{array}$ & $\mathrm{N}$ & $\begin{array}{c}\text { Boluses } \\
\text { (Day 1) }\end{array}$ & Distance & $\begin{array}{c}\text { Time } \\
\text { in } \\
\text { Contact }\end{array}$ \\
\hline Bottle & 8 & $3.4 \mathrm{ab}$ & $24.1_{\mathrm{a}}$ & $5 \%_{\mathrm{a}}$ \\
Food & 8 & $3.7_{\mathrm{ab}}$ & $21.3^{*} \mathrm{a}$ & $8 \%_{\mathrm{a}}$ \\
Clear Hole & 8 & $6.5 \mathrm{a}_{\mathrm{a}}$ & $19.7 \mathrm{t}_{\mathrm{a}}$ & $12 \%_{\mathrm{a}}$ \\
Dark Hole & 8 & $3.7_{\mathrm{ab}}$ & $17.1 \dagger_{\mathrm{a}}$ & $30 \%_{\mathrm{b}}$ \\
Rat & 8 & $2.1_{\mathrm{b}}$ & $9.2+_{\mathrm{b}}$ & $62 \%_{\mathrm{c}}$ \\
Mean & 40 & 3.9 & 18.3 & $24 \%$ \\
Novel Object & 10 & 2.7 & 18.8 & $23 \%$ \\
\hline
\end{tabular}

Note-Entries with a common subscript are not significantly different from each other

*Significantly lower than chance distance by two-tailed $t$ tests, $p<.01$ 


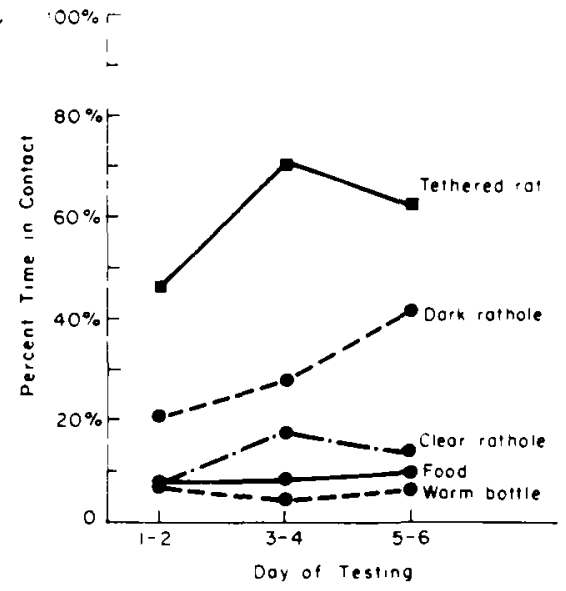

Fig. 1. Time in contact as a function of stimulus object and day of testing.

independent of shade seeking, eight rats were tested for 6 days with a clear Plexiglas tube, identical to the dark rathole, but unpainted.

Tethered Rat

In order to provide a baseline against which to evaluate attraction to the various nonsocial objects, eight rats were tested for 6 days with a stimulus rat of the same age and sex as the S. Stimulus rats were tethered by a harness of fine fishline looped around their shoulders and tacked to the floor of the open field. They were free to react to the $S$ rat, rear $u p$, and move about within their own body lengths but could not, of course, engage in gross locomotion about the open field. Novel Stimulus

In each of the preceding conditions, rats were tested with the same stimulus object each day. It is possible that one of the reasons rats are attracted to other rats is that they provide a continuously changing unpredictable stimulus object. If rats are attracted to sheer novelty, it should be possible to show that they will be attracted to novel nonsocial objects. In the present experiment, 10 rats were tested with a different one of the various stimulus objects described above each day for 5 days. Objects were presented in counterbalanced order such that each object was presented equally often on each day and each object was followed by every other object an equal number of times. On the sixth day of testing, each rat was exposed to the same object it had encountered on the fifth.

\section{Procedure}

Rats were tested daily for $5 \mathrm{~min}$ in the open field. Os recorded the amount of time each animal spent in direct physical contact with the various stimulus objects and recorded the location of the animals at $10 \mathrm{sec}$ intervals signaled by a clicker. The average distance in inches between rat and stimulus objects was later calculated from these location scores. After each test period, fecal boluses were counted and removed and the field wiped with a sponge dampened in a vinegar-water solution.

\section{RESULTS}

Rats were much more attracted to a tethered rat than to the various nonsocial objects. They spent an average of $62 \%$ of their time in the open field in direct physical contact with the tethered rat, as compared to only $14 \%$ with the various objects $(\mathrm{p}<.001)^{1}$ and maintained an average distance of only $9.2 \mathrm{in}$. from the rat, as compared to $20.1 \mathrm{in}$. in the other conditions $(p<.001)$. There was virtually no overlap in attraction scores between the social and nonsocial conditions.

The nonsocial objects differed among themselves in attractiveness (Table 1). Rats were least attracted to the warm bottle, and in fact, stayed no closer to it than would be expected by chance. Rats were slightly but significantly attracted to the food and clear tube, as compared to the chance baseline of 25.2 in. calculated as if rats paid no attention to the object.

The dark rathole was the only nonsocial object to elicit any substantial interest, with rats spending an average of $30 \%$ of their time in the field inside it or touching the outside. There was considerable variation among the rats in the attraction to the rathole, much higher than in attraction to the other objects $(\mathrm{p}<.01$ by variance ratio). Some rats were very much attracted to the dark rathole, spending over two-thirds of their time near it, while other rats showed almost no attraction at all.

There was an overall effect of day of testing $(p<.001)$ and an interaction with stimulus object ( $p<.001)$ such that rats became more attracted to the tethered rat and to the dark rathole over days but not to the other stimulus objects (Fig. 1). Similar effects have been found in previous research (Latané, 1969; Latané \& Glass, 1968) and probably reflect reduced exploration as rats become familiar with the open field (Eckman, Meltzer, \& Latané, 1969).

Novelty did not enhance the attractiveness of these objects. Rats exposed to a different object each day spent an average of $23 \%$ of their time in contact as compared to $24 \%$ for rats exposed to the same object (n.s.) and maintained an average distance of 18.8 in. as compared to 18.3 in. (n.s.). Each of the objects elicited comparable degrees of attraction, whether it was novel or familiar. It appears that novelty, within the limits tested here, does not affect attraction.

Rats were more attracted to another rat than to the various nonsocial objects, and they also defecated less in this condition, even on the first day ( 2.1 boluses vs $4.4, p<.02$ ). This is consistent with findings by Latané (1969) and Latané \& Glass (1968) that rats in pairs show less emotionality than singly tested rats.

\section{DISCUSSION}

Rats were much more attracted to other rats than to any of a variety of nonsocial objects, each chosen to provide possible sources of satisfaction, such as warmth, secondary reinforcement, or shade. It is clear that no one of these nonsocial satisfactions provides a complete explanation of why rats are attracted to one another.

It is possible that rats are more attractive than objects because they offer a combination of several or all of these satisfactions. It also may be that the various objects had unknown repellant features that combined with their otherwise attractive qualities to reduce approach. It seems more likely, however, that rats are not primarily attracted to other rats for these reasons but rather because they enjoy the opportunity for rewarding social interaction.

\section{REFERENCES}

ANTONITIS, J. J., \& SHER, A. J. Social regression in the white rat. Journal of Psychology, 1952, 33, 99-111.

CAPPELL, H. \& LATANE, B. Effects of alcohol and caffeine on the social and emotional behavior of the rat. Quarterly Journal of Studies in Alcohol, 1969, 30, 345-356.

ECKMAN, J., MELTZER, J., \& LATANÉ B. Gregariousness in rats as a function of familiarity of environment. Journal of Personality \& Social Psychology. 1969. $11,107-114$

LATANÉ, B. Gregariousness and fear in laboratory rats. Journal of Experimental Social Psychology, 1969, 5, 61-69.

LATANE, B., CAPPELL, H., \& JOY, V Social deprivation, housing density and gregariousness in rats. Journal of Comparative \& Physiological Psychology, $1970,70,221-227$.

LATANÉ, B., \& GLASS, D. C. Social and nonsocial attraction in rats. Journal of Personality \& Social Psychology, 1968, 9. 142-146.

\section{NOTE}

1. All $p$ values based on analysis of variance or two-tailed tests, unless otherwise noted. 